\title{
Black-Scholes Fuzzy Numbers as Indexes of Performance
}

\author{
M. R. Simonelli \\ Dipartimento di Statistica e Matematica per la Ricerca Economica, Università di Napoli Parthenope, \\ Via Acton, 38, 80133 Naples, Italy \\ Correspondence should be addressed to M. R. Simonelli, mariarosaria.simonelli@uniparthenope.it
}

Received 10 June 2009; Accepted 29 January 2010

Academic Editor: Antonio Di Nola

Copyright (C) 2010 M. R. Simonelli. This is an open access article distributed under the Creative Commons Attribution License, which permits unrestricted use, distribution, and reproduction in any medium, provided the original work is properly cited.

\begin{abstract}
We use the set of propositions of some previous papers to define a fuzzy version of the Black-Scholes value where the risk free instantaneous interest intensity, the volatility and the initial stock price are fuzzy numbers whose parameters are built with statistical financial data. With our Black-Scholes fuzzy numbers we define indexes of performance varing in time. As an example, with data of the Italian Stock Exchange on MIB30, we see that in 2004 and 2006 our indexes are negative, that is, they are indexes of the refuse to invest and this refuse increased. So, on November 11, 2006 we could forecast that the market will become with more risk: the risk of loss will increase. Now, on January 25, 2010, we know that this forecast has happened. Obviously, the parameters of our Black-Scholes fuzzy numbers can be valued also with incomplete, possibilistic data. With respect to the probabilistic one, our fuzzy method is more simple and immediate to have a forecast on the financial market.
\end{abstract}

\section{Introduction}

In this paper, we use the fuzzy theory of Zadeh [1], the set of propositions used in some previous papers of us [2-4] and statistical data, to have a fuzzy version of the BlackScholes (B-S) value for a European call option, where the istantaneous intensity of the risk-free interest, the standard deviation of the instantaneous intensity of return from the underlying security (volatility), and the initial stock price are fuzzy numbers built with statistical financial data. Obviously, a similar method can be used if one has incomplete possibilistic data.

With a theorem we deduce that our B-S fuzzy sets are fuzzy numbers.

Then we give a statistical example: with the data of the Italian MIB 30, that is, with its time series, we build a $B-S$ fuzzy number which for every belief degree gives a B-S value.

With the support of our B-S fuzzy numbers we define indexes of the performance of the market varing in time. In particular, in 2004 and 2006 we see that these indexes are negative, that is, they are indexes of the refuse to invest. So, comparing our indexes of 2004 and 2006, on November 11, 2006 we could forecast for future loss in the gamble on the increase of the MIB30. The forecast happened.
This paper is organized as follows. In Section 2 we set the preliminaries and give the theorems which link the Zadeh's extension principle to the construction of fuzzy numbers with cut-functions in accordance with the theorems in $[2,3,5]$ and Zadeh's identity; inSection 3 we define three fuzzy numbers which we deduce by probabilistic densities to use for our applications; in Section 4 we give our model of the Black-Scholes fuzzy numbers and its theorem; and in Section 5 we put the applications and cues for further researchs.

\section{Preliminaries and Theorems}

Let $(\Omega, \mathcal{A})$, be a probabilizable space, where $\Omega$ is the set of real numbers, $\mathcal{A}=\mathscr{B}(\Omega)$ is the Borel's $\sigma$-field.

We may identify a subset $A$ of $\Omega$ (event) with its characteristic function,

$$
\chi_{A}: \omega \in \Omega \longrightarrow \chi_{A}(\omega) \in\{0,1\},
$$

where $A$ is the support of $\chi_{A}$ :

$$
\chi_{A}(\omega)= \begin{cases}1, & \omega \in A ; \\ 0, & \omega \notin A .\end{cases}
$$


A fuzzy subset $A$ (fuzzy event) is identified with its membership function, $f$, (see Zadeh [1]):

$$
f: \omega \in \Omega \longrightarrow f(\omega) \in[0,1],
$$

that is, $0 \leq f(\omega) \leq 1$; the support of $f$ is $\tilde{A}=\{\omega \in \Omega$ : $f(\omega) \neq 0\} ; f(\omega)$ represents (i) a membership degree of $\omega$ as element of $\widetilde{A}$; (ii) a belief degree that $\omega$ happens.

The $y$-cut of $f$, stated at the level $y \in[0,1]$, is defined by:

$$
C_{y}^{f}=\{\omega \in \Omega: f(\omega) \geq y\} .
$$

We say that $f$ is a fuzzy number if $f$ is convex, that is, all its $y$-cuts are closed and bounded real intervals; $f$ is normal, that is, there exists $\omega_{0} \in \Omega$ such that $f\left(\omega_{0}\right)=1 ; f$ is upper semi-continuous (usc); $f$ is compactly supported on the real line. We denote by $\mathcal{N}$ the set of fuzzy numbers.

If $f \in \mathcal{N}$ then

$$
\forall y \in[0,1], \quad C_{y}^{f}=\left[f^{-l}(y), f^{-r}(y)\right],
$$

where $f^{-l}$ is increasing and is called the left cut-function of $f, f^{-r}$ is decreasing and is called the right cut-function of $f$. $f^{-l}, f^{-r}$ are left-continuous because $f \in \mathcal{N}$ (see [2, page 5]). In this case, we have the following Zadeh's identity:

$$
f(\omega)=\sup _{y \in[0,1]} y \cdot \chi_{\left[f^{-l}(y), f^{-r}(y)\right]}(\omega),
$$

where $\chi$ is the characteristic function of the real interval $\left[f^{-l}(y), f^{-r}(y)\right]$.

Zadeh's Extension Principle. Let $x:=\left(x_{1}, \ldots, x_{n}\right) \in R^{n}, h(x)$ a continuous deterministic function with values in $\Omega$ :

$$
\begin{gathered}
h: x \in R^{n} \longrightarrow h(x)=\omega \in \Omega, \\
f_{1}, f_{2}, \ldots, f_{n},
\end{gathered}
$$

let $h$ be a deterministic function and $f_{1}, f_{2}, \ldots, f_{n}, n$ be fuzzy numbers. The Zadeh's extension principle (Z.e.pr.) gives a belief degree to every value $\omega=h(x)$, that is, the Z.e.pr. defines a belief degree or membership function, $\widetilde{h}$, by

$$
\tilde{h}(\omega):=\sup _{x \in R^{n}: h(x)=\omega} \min \left\{f_{1}\left(x_{1}\right), \ldots, f_{n}\left(x_{n}\right)\right\} .
$$

So, from the deterministic function $h$, we have that the Z.e.pr. induces a fuzzy number, that is,

$$
\tilde{h}: \omega \longrightarrow \tilde{h}(\omega) \in[0,1]
$$

and $\tilde{h}$ is obtained by the deterministic function $h$ and the fuzzy numbers $f_{1}, \ldots, f_{n}$, that is,

$$
\left(f_{1}, \ldots, f_{n}\right) \in \mathcal{N}^{n} \longrightarrow \tilde{h} \in \mathcal{N} .
$$

Proposition 1. If $f_{1}, \ldots, f_{n}$ are in $\mathcal{N}$ and $h$ is a continuous deterministic function, then (for all $y \in[0,1]$ ) the $y$-cut of the fuzzy number $\tilde{h}\left(f_{1}, \ldots, f_{n}\right)$ is equal to the $h$ of the $y$-cuts of $f_{1}, \ldots, f_{n}$ :

$$
\begin{aligned}
C_{y}^{h\left(f_{1}, \ldots, f_{n}\right)} & =h\left(C_{y}^{f_{1}}, \ldots, C_{y}^{f_{n}}\right) \\
& =\left\{\omega=h\left(x_{1}, \ldots, x_{n}\right) \in \Omega: x_{1} \in C_{y}^{f_{1}}, \ldots, x_{n} \in C_{y}^{f_{n}}\right\} .
\end{aligned}
$$

This Proposition is an extension obtained by induction by a Proposition of Biacino-Lettieri (see [5, Section 2.4)] and it is used in our previous papers [2-4].

From Proposition 1 we have the following proposition.

Proposition 2. Let $h\left(x_{1}, \ldots, x_{n}\right)$ be a continuous deterministic function increasing with respect to $x_{i}$ and decreasing with respect to $x_{j}$ and let $f_{1}, \ldots, f_{n}$ be in $\mathcal{N}$, then we have that the membership function $\tilde{h}$ is in $\mathcal{N}$ if we have that

(i) the left cut-function $\tilde{h}^{-l}(y), y \in[0,1]$ is given by

$$
\tilde{h}^{-l}(y)=h\left(\ldots, f_{i}^{-l}(y), \ldots, f_{j}^{-r}(y), \ldots\right)
$$

and is increasing;

(ii) the right cut-function $\tilde{h}^{-r}(y), y \in[0,1]$ is given by

$$
\tilde{h}^{-r}(y)=h\left(\ldots, f_{i}^{-r}(y), \ldots, f_{j}^{-l}(y), \ldots\right)
$$

and is decreasing;

(iii) for all $y \in[0,1], \tilde{h}^{-l}(y) \leq \tilde{h}^{-r}(y)$ and the equal holds if $y=1$.

Then the membership function $\tilde{h}$ has the following shape

$$
\tilde{h}(\omega)=\max _{y \in[0,1]} y \cdot \chi_{\left[\tilde{h}^{-l}(y), \tilde{h}^{-r}(y)\right]}(\omega)
$$

and it is an element of $\mathcal{N}$, where $\chi$ is the characteristic function of the real interval $\left[\tilde{h}^{-l}(y), \tilde{h}^{-r}(y)\right]$.

Proof. If $f_{1}, \cdot, f_{n} \in \mathcal{N}$ then we have that for all $y \in[0,1]$ their cuts are the following compact (closed and bounded) intervals, $i \in\{1, \ldots n\}$ :

$$
C_{y}^{f_{i}}=\left[f_{i}^{-l}(y), f_{i}^{-r}(y)\right] .
$$

On the other hand, by Proposition 1, from the hypothesis of continuity of $h$ it follows that $C_{y}^{\tilde{h}\left(f_{1}, \cdot, f_{n}\right)}$ is a compact interval. So, if the left cut-function $\widetilde{h}^{-l}(y)$ is increasing and the right $\tilde{h}^{-r}(y)$ is decreasing, $\tilde{h}^{-l}(y) \leq \tilde{h}^{-r}(y)$ and $\tilde{h}^{-l}(1)=\tilde{h}^{-r}(1)$, then $C_{y}^{\tilde{h}}$ is equal to the following

$$
C_{y}^{\tilde{h}}=\left[\tilde{h}^{-l}(y), \tilde{h}^{-r}(y)\right],
$$

that is $\tilde{h}$ is in $\mathcal{N}$ and by Zadeh's identity we have that $\tilde{h}$ has the following shape:

$$
\tilde{h}(\omega)=\max _{y \in[0,1]} y \cdot \chi_{\left[\tilde{h}^{-l}(y), \tilde{h}^{-r}(y)\right]}(\omega) .
$$




\section{Examples of Fuzzy Numbers Useful for Our Applications}

For our option price fuzzy numbers we can use, for example, one of the following fuzzy numbers.

(1) Triangular fuzzy numbers

$$
f(\omega)= \begin{cases}\frac{\omega-a}{b-a}, & \text { if } \omega \in[a, b] \\ \frac{c-\omega}{c-b}, & \text { if } \omega \in[b, c] \\ 0, & \text { elsewhere }\end{cases}
$$

where $a<b<c$. The graph of $f$ looks like a triangle. In our cases, the support $[a, c]$ may be calculated by a statistical time series and $b$ may be equal to its arithmetical mean or the value of the time series with the bigger frequence, but also with possibilistic data.

In this case the $y$-cuts are given by

$$
\forall y \in] 0,1], \quad\left[f^{-l}(y), f^{-r}(y)\right]
$$

with

$$
\begin{aligned}
& f^{-l}(y)=a+(b-a) y ; \\
& f^{-r}(y)=c-(c-b) y .
\end{aligned}
$$

(2) Gaussian fuzzy set

$$
g(\omega)=e^{-(\omega-\mu)^{2} / 2 \sigma^{2}},
$$

where the mean $\mu$ and the standard deviation $\sigma$ are calculated using a statistical time series.

For all $y \in] 0,1]$ the cut-functions are given by

$$
\begin{aligned}
& g^{-l}(y)=\mu-\sqrt{-2 \sigma^{2} \log _{e}(y)}, \\
& g^{-r}(y)=\mu+\sqrt{-2 \sigma^{2} \log _{e}(y) .}
\end{aligned}
$$

The belief degree of $\omega=\mu$ is equal to $g(\mu)=1$ which is the max of $g$. In fact the derivative of $g$ is given by

$$
g^{\prime}(\omega)=\left(\frac{-(\omega-\mu)}{\sigma^{2}}\right) e^{-(\omega-\mu)^{2} / 2 \sigma^{2}}
$$

which is equal to zero if $\omega=\mu$. The function $g$ has a symmetric and campanulate graph.

The flex points are $\mu-\sigma, \mu+\sigma$. In fact the second derivative of $g$ is given by

$$
\begin{aligned}
g^{\prime \prime}(\omega) & =-\frac{1}{\sigma^{2}} e^{-(\omega-\mu)^{2} / 2 \sigma^{2}}-\frac{\omega-\mu}{\sigma^{2}}\left(\frac{-(\omega-\mu)}{\sigma^{2}}\right) e^{-(\omega-\mu)^{2} / 2 \sigma^{2}} \\
& =-\frac{1}{\sigma^{2}} e^{-(\omega-\mu)^{2} / 2 \sigma^{2}}\left[1-\frac{(\omega-\mu)^{2}}{\sigma^{2}}\right],
\end{aligned}
$$

$g^{\prime \prime}(\omega)=0$ if and only if $\left(1 / \sigma^{2}\right)(\omega-\mu)^{2}=1$, that is, if $(\omega-\mu)^{2}=\sigma^{2} \Leftrightarrow \omega-\mu= \pm \sigma \Leftrightarrow \omega=\mu \pm \sigma$. The function $g$ is not in $\mathcal{N}$ because its support is not compact.
(3) Gaussian fuzzy number (with compact support)

$g(\omega)=\left\{\begin{array}{l}e^{-(\omega-\mu)^{2} / 2 \sigma^{2}}, \quad \text { if } \omega \in[\mu-3.9 \sigma, \mu+3.9 \sigma] \\ 0, \quad \text { elsewhere, }\end{array}\right.$

where $\mu$ and $\sigma$ are calculated with the statistical time series or with possibilistic data. We choose 3.9 because the statistical tables are up to this number; the support of $g$ is $[\mu-3.9 \sigma, \mu+$ $3.9 \sigma$ ] which influences the results. We can use, for example, 4.1 to have a larger support $[\mu-4.1 \sigma, \mu+4.1 \sigma]$.

\section{Black-Scholes Fuzzy Numbers}

Let $k$ be the strike price, $\delta$ the annual istantaneous intensity of the risk-free interest, $a$ the initial market price of the underlying security, $t$ the expiration date, $\sigma^{2}$ the variance of the annual istantaneous intensity of the random return from the underlying security, then the classical Black-Scholes value for a European call option at time zero is given by

$$
c(a, \delta, \sigma)=a N\left(d_{1}\right)-k e^{-\delta t} N\left(d_{2}\right),
$$

where $N(x)$ is the cumulative distribution function for a standard normal random variable (r.v.):

$$
N(x)=\frac{1}{\sqrt{2 \pi}} \int_{-\infty}^{x} e^{-(1 / 2) \omega^{2}} d \omega
$$

also

$$
d_{1}=\frac{\log (a / k)+\left(\delta+(1 / 2) \sigma^{2}\right) t}{\sigma \sqrt{t}}
$$

and $d_{2}=d_{1}-\sigma \sqrt{t}$.

Note that if the volatility $\sigma$ is very big then we may have $d_{1}>3.9$ hence $d_{2}<-3.9$. In this case the values of the cumulative distribution function of the standard normal r.v. become $N\left(d_{1}\right)=1, N\left(d_{2}\right)=0$, respectively, and so we have that the price of the call is equal to the price of the underlying security at time zero (this is the extreme superior value of the call) (see $[6,7])$.

The price of the European call (26) depends in particular on the initial market price of the underlying security, $a$, and on the strike price, $k$. If we consider (26) varing in time, then its fuzzy versions give us indexes of performance as we see in Proposition 3 and Section 5 where we make a statistical application on MIB30.

Proposition 3 (on black-scholes fuzzy numbers). If we take these fuzzy numbers

$$
f_{1}=\tilde{a}, \quad f_{2}=\tilde{\delta}, \quad f_{3}=\tilde{\sigma} \in \mathcal{N}
$$

instead of the corresponding crisp ones, then the fuzzy version of the Black-Scholes formula, $\tilde{h}=\tilde{c}$, is in $\mathcal{N}$ if and only if

(i) the left cut-function of $\tilde{c}$ given by

$$
\begin{aligned}
& \tilde{c}^{-l}(y)=\tilde{a}^{-l}(y) N\left(\tilde{d}_{1}^{-l}(y)\right)-k e^{-t \tilde{\delta}^{-l}(y)} N\left(\tilde{d}_{2}^{-r}(y)\right) \\
& \text { is increasing; }
\end{aligned}
$$


(ii) the right cut-function of $\tilde{c}$ given by

$$
\tilde{c}^{-r}(y)=\tilde{a}^{-r}(y) N\left(\tilde{d}_{1}^{-r}(y)\right)-k e^{-t \tilde{\delta}^{-r}(y)} N\left(\tilde{d}_{2}^{-l}(y)\right)
$$

is decreasing;

(iii) the left cut-function of $\tilde{c}$ is less than the right:

$$
\tilde{c}^{-l}(y) \leq \tilde{c}^{-r}(y) .
$$

Moreover, with the left and right cut-functions we may write $\tilde{c}$ :

$$
\tilde{c}(\omega)=\sup _{y \in[0,1]} y \cdot \chi_{\left[c^{-l}(y), c^{-r}(y)\right]}(\omega),
$$

and $\tilde{c}$ is an element of $\mathcal{N}$.

Proof. We suppose to know these three fuzzy numbers

$$
f_{1}=\tilde{a}, \quad f_{2}=\tilde{\delta}, \quad f_{3}=\tilde{\sigma} \in \mathcal{N},
$$

that is, for all $x=\left(x_{1}, x_{2}, x_{3}\right) \in R^{3}$, we suppose to know the belief degree $0 \leq \tilde{a}\left(x_{1}\right) \leq 1$ of the fuzzy event $\left\{a=x_{1}\right\}$ and analogously we know the belief degree $0 \leq \tilde{\delta}\left(x_{2}\right) \leq 1$ and $0 \leq \tilde{\sigma}\left(x_{3}\right) \leq 1$ of the fuzzy events $\left\{\delta=x_{2}\right\}$ and $\left\{\sigma=x_{3}\right\}$, respectively.

Then we obtain the fuzzy price of the call, $\tilde{c}$ by the Zadeh's extension principle:

$$
\tilde{c}(\omega):=\sup _{x \in R^{3}: c(x)=\omega} \min \left\{\tilde{a}\left(x_{1}\right), \tilde{\delta}\left(x_{2}\right), \tilde{\sigma}\left(x_{3}\right)\right\}
$$

according to the Black-Scholes formula

$$
c(a, \delta, \sigma)=a N\left(d_{1}\right)-k e^{-\delta t} N\left(d_{2}\right)
$$

On the other hand, if we know the fuzzy numbers $\tilde{a}, \tilde{\delta}, \tilde{\sigma} \in \mathcal{N}$ then we can write their left and right cut-functions

$$
\forall y \in[0,1], \quad \begin{array}{ll}
\tilde{a}^{-l}(y), \tilde{\delta}^{-l}(y), \tilde{\sigma}^{-l}(y) ; \\
\tilde{a}^{-r}(y), \tilde{\delta}^{-r}(y), \tilde{\sigma}^{-r}(y) .
\end{array}
$$

Taking into account that $d_{1}$ has $\sigma$ at the denominator, we have that for every fixed belief degree, $y \in[0,1]$, the cut functions of the fuzzy numbers $\tilde{d}_{1}$ and $\tilde{d}_{2}$ are given by

$$
\begin{gathered}
\tilde{d}_{1}^{-l}(y)=\frac{\log \left(\tilde{a}^{-l}(y) / k\right)+\left[\tilde{\delta}^{-l}(y)+(1 / 2)\left(\tilde{\sigma}^{-l}(y)\right)^{2}\right] t}{\tilde{\sigma}^{-r}(y) \sqrt{t}} ; \\
\tilde{d}_{2}^{-l}(y)=d_{1}^{-l}(y)-\tilde{\sigma}^{-r}(y) \sqrt{t} ; \\
\tilde{d}_{2}^{-r}(y)=\frac{\log \left(\tilde{a}^{-r}(y) / k\right)+\left[\tilde{\delta}^{-r}(y)+(1 / 2)\left(\tilde{\sigma}^{-r}(y)\right)^{2}\right] t}{\tilde{\sigma}^{-l}(y) \sqrt{t}} ;
\end{gathered}
$$

In this way, the left cut-functions are increasing and the right cut-functions are decreasing.
Since $c$ is increasing with respect to $a, d_{1}$, and $\delta$ and decreasing with respect to $d_{2}$, by Proposition 2 , we have that the left and right cut-functions of $\tilde{c}$ are given by

$$
\begin{aligned}
& \tilde{c}^{-l}(y)=\tilde{a}^{-l}(y) N\left(\tilde{d}_{1}^{-l}(y)\right)-k e^{-t \tilde{\delta}^{-l}(y)} N\left(\tilde{d}_{2}^{-r}(y)\right), \\
& \tilde{c}^{-r}(y)=\tilde{a}^{-r}(y) N\left(\tilde{d}_{1}^{-r}(y)\right)-k e^{-t \tilde{\delta}^{-r}(y)} N\left(\tilde{d}_{2}^{-l}(y)\right) .
\end{aligned}
$$

Moreover we have $\tilde{c}^{-l}(y) \leq \tilde{c}^{-r}(y)$ and by Zadeh's identity

$$
\widetilde{c}(\omega)=\sup _{y \in[0,1]} y \cdot \chi_{\left[c^{-l}(y), c^{-r}(y)\right]}(\omega) .
$$

For $y=0$, we have that the support of the fuzzy Black-Schose number $\tilde{c}$ is

$$
\Omega=\left[\tilde{c}^{-l}(0), \tilde{c}^{-r}(0)\right]
$$

In particular we have that the real number $\tilde{c}^{-l}(0)$ is negative if $\tilde{a}^{-l}(0) \ll k$ and $\tilde{c}^{-r}(0)$ is positive if $\tilde{a}^{-r}(0) \gg k$. The two prices

$$
\tilde{c}^{-l}(0), \quad \tilde{c}^{-r}(0)
$$

represent the pessimistic and optimistic performance on the future if we gamble on the increase of the price.

The real numbers $\tilde{c}^{-l}(0), \tilde{c}^{-r}(0)$ are two synthesis of the fuzzy parameters which we use, so they may represent indexes of the performance of the market of the underlying security. Obviously if the underlying security is a market index, as the MIB30, then $\tilde{c}^{-l}(0), \tilde{c}^{-r}(0)$ may represent indexes of the performance of the market. Obviously these indexes vary in time. We will see that their variations help us to evaluate and to forecast the future of the market.

\section{Fuzzy Numbers with Parameters Valued with Statistical Observations}

Here we build a fuzzy set using statistical observations of financial prices. We have a time series of the daily prices,

$$
p(t)
$$

of the "MIB 30" from the first of April 2001 to the first of October 2006 (for 67 months) and we want to build a fuzzy option price for an option, of the first of October 2006, with strike price 39500 , maturity on 11 days.

From the security time series of the daily price we build the time series of the instantaneous intensity of returns from the security (using continuous compounding),

$$
r(t)=\log _{e} \frac{p(t)}{p(t-1)} .
$$

Every month we have a daily arithmetical mean of $r(t)$. So, from the observation of 67 months we have 67 means:

$$
\forall k \in\{1,2, \ldots, 67\}, \quad m(k)=\frac{1}{30} \sum_{t=1}^{30} r(t)
$$


and 67 daily arithmetical variances of $r_{t}$ :

$$
\forall k \in\{1,2, \ldots, 67\}, \quad s^{2}(k)=\frac{1}{29} \sum_{t=1}^{30}(r(t)-m(k))^{2} .
$$

So we have a time series of 67 standard deviations, that is, the volatility time series:

$$
\forall k \in\{1,2, \ldots, 67\}, \quad s(k)=\sqrt{s^{2}(k)} .
$$

From this time series we can build a fuzzy number, $\tilde{\sigma}$, to represent the fuzzy volatility. We can do this in many ways. One of these is to calculate the arithmetical mean and the variance of the volatility time series:

$$
\begin{gathered}
\mu=\frac{1}{67} \sum_{k=1}^{67} s(k), \\
\sigma=\frac{1}{67} \sum_{k=1}^{67}(s(k)-m(s))^{2}
\end{gathered}
$$

and to take the Gaussian fuzzy number with mean $\mu$ and variance $\sigma^{2}$.

Another way is using a triangular fuzzy number to represent the fuzzy volatility. In this case we need to know the support. We can do this reading the time series. In the case of the "MIB 30" volatility we have

(1) the min variance of the volatility in August 2006: 0.000068 ;

(2) the max daily variance of the volatility in September 2002: 0.000521 .

We have to multiply by 365 for obtaining the min variance per annum:

$$
0.000068 \cdot 365=0.02482
$$

and the max variance per annum:

$$
0.000521 \cdot 365=0.190165 \text {. }
$$

The min and max standard deviations per annum were

$$
\sqrt{0.02482}=0.1575, \quad \sqrt{0.190165}=0.436079,
$$

respectively.

So we have the interval $[0.1575,0.436079]$, but if we think that the standard deviation has in the interval $[0.15,0.50]$ and the value with more belief degree is 0.25 , we may take as triangular fuzzy annual volatility of the "MIB 30 ", the following fuzzy number:

$$
\tilde{\sigma}(\omega)= \begin{cases}\frac{\omega-0.15}{0.10}, & \text { if } \omega \in[0.15,0.25] \\ \frac{0.50-\omega}{0.25}, & \text { if } \omega \in[0.25,0.50] \\ 0, & \text { elsewhere. }\end{cases}
$$

The $y$-cuts are given by

$$
\begin{gathered}
\forall y \in[0,1], \quad\left[\tilde{\sigma}^{-l}(y), \tilde{\sigma}^{-r}(y)\right] \\
\tilde{\sigma}^{-l}(y)=0.15+0.10 y ; \tilde{\sigma}^{-r}(y)=0.50-0.25 y .
\end{gathered}
$$

So with belief degree $y=0.90$ we have that the annual volatility is in the real interval $C_{y}^{\tilde{\sigma}}=[0.24,0.275]$ because

$$
\begin{gathered}
\tilde{\sigma}^{-l}(0.90)=0.15+0.10(0.90)=0.24, \\
\tilde{\sigma}^{-r}(0.90)=0.50-0.25(0.90)=0.275 .
\end{gathered}
$$

For building the fuzzy istantaneous intensity of the riskfree interest rate for the same duration of the option, we read

(1) the risk-free interest rates of the Italian Banks in the real interval $i_{1} \in[0.015,0.018]$ then it follows that $\delta_{1} \in[0.0149,0.017]$;

(2) the interest rates of the "Buoni Ordinari del Tesoro" $i_{2} \in[0.02,0.025] \Rightarrow \delta_{2} \in[0.0198,0.02469]$.

So, we give a great belief degree to these data if we take the basic points of the triangle in this way: $b=\widetilde{\delta}^{l}(1)=$ $0.02, a=0.014=\widetilde{\delta}^{l}(0), c=0.025=\widetilde{\delta}^{r}(0)$ so the support of the triangular fuzzy number $\tilde{\delta}$ is the interval $[0.014,0.025]$ :

$$
\widetilde{\delta}(\omega)= \begin{cases}\frac{\omega-0.014}{0.006}, & \text { if } \omega \in[0.014,0.02] \\ \frac{0.025-\omega}{0.005}, & \text { if } \omega \in[0.02,0.025] \\ 0, & \text { elsewhere. }\end{cases}
$$

The $y$-cut functions are $\widetilde{\delta}^{-l}(y)=0.014+0.006 y ; \widetilde{\delta}^{-r}(y)=$ $0.025-0.005 y$.

On October 30, 2006 the "MIB 30" had the following prices: the last price 39500 , the max 40000 and min 38000 . We can use these prices to build the triangular fuzzy number, $\tilde{a}$ in this way:

$$
\tilde{a}(\omega)= \begin{cases}\frac{\omega-38000}{1500}, & \text { if } \omega \in[38000,39500] ; \\ \frac{40000-\omega}{500}, & \text { if } \omega \in[39500,40000] ; \\ 0, & \text { elsewhere. }\end{cases}
$$

The $y$-cut functions are

$$
\begin{aligned}
& \tilde{a}^{-l}(y)=38000+1500 y, \\
& \tilde{a}^{-r}(y)=40000-500 y .
\end{aligned}
$$

With belief degree $y=1$ we have that our MIB30 is equal to $\tilde{a}^{-l}(1)=\tilde{a}^{-r}(1)=39500$. The values 38000 and 40000 have membership degrees equal to zero.

But if we want to make evaluations on the future we may say that in the future with belief degree $y=0.5$ the min and max price of MIB30 will be equal to

$$
\begin{aligned}
& \tilde{a}^{-l}(0.50)=38000+1500 \cdot 0.50=38750 ; \\
& \tilde{a}^{-r}(0.50)=40000-500 \cdot 0.50=39750 .
\end{aligned}
$$


Statistical Fuzzy Black-Scholes. Using the triangular fuzzy numbers $\tilde{a}, \tilde{\delta}, \tilde{\sigma}$, built with statistical data, we may write the cut functions of the triangular fuzzy Black-Scholes, $\tilde{c}$, on "MIB 30 with strike price 39000, maturity on 11 days".

The left cut-function of $\tilde{c}$ is given by

$$
\tilde{c}^{l}(y)=\tilde{a}^{l}(y) N\left(\tilde{d}_{1}^{l}(y)\right)-39000 e^{-(11 / 365) \tilde{\delta}^{l}(y)} N\left(\tilde{d}_{2}^{r}(y)\right),
$$

where

$$
\begin{aligned}
& \tilde{d}_{1}^{l}(y) \\
& =\frac{\log \left(\tilde{a}^{l}(y) / 39000\right)+\left[\tilde{\delta}^{l}(y)+(1 / 2)\left(\tilde{\sigma}^{l}(y)\right)^{2}\right](11 / 365)}{\tilde{\sigma}^{r}(y) \sqrt{11 / 365}}
\end{aligned}
$$

and $\tilde{d}_{2}^{l}(y)=d_{1}^{l}(y)-\tilde{\sigma}^{r}(y) \sqrt{11 / 365}$;

The right cut-function of $\tilde{c}$ is

$$
\tilde{c}^{r}(y)=\tilde{a}^{r}(y) N\left(\tilde{d}_{1}^{r}(y)\right)-39000 e^{-t \tilde{\delta}^{r}(y)} N\left(\tilde{d}_{2}^{l}(y)\right),
$$

where

$$
\tilde{d}_{1}^{r}(y)=\frac{\log \left(\tilde{a}^{r}(y) / 39000\right)+\left[\tilde{\delta}^{r}(y)+\left(\tilde{\sigma}^{r}(y)\right)^{2} / 2\right] 0.03}{\tilde{\sigma}^{l}(y) \sqrt{0.03}},
$$

and $\tilde{d}_{2}^{r}(y)=\tilde{d}_{1}^{r}(y)-\tilde{\sigma}^{l}(y) \sqrt{0.03}$.

Now we have to calculate the basic point of the triangle: the min value of the call with belief degree zero, $\tilde{c}^{l}(0)$, the mean value with belief degree $1, \tilde{c}^{l}(1)$, and the max value of the call with belief degree zero $\tilde{c}^{r}(0)$.

The value of the cut function with the max belief degree, $\tilde{c}^{l}(1)=c^{r}(1)$, is given by

$$
\tilde{c}^{l}(1)=\tilde{a}^{l}(1) N\left(\tilde{d}_{1}^{l}(1)\right)-39000 e^{-11 / 365 \tilde{\delta}^{l}(1)} N\left(\tilde{d}_{2}^{r}(1)\right) .
$$

So we have to calculate $\tilde{d}_{1}^{l}(1)=\tilde{d}_{1}^{r}(1)$ and $\tilde{d}_{2}^{r}(1)$ :

$$
\begin{aligned}
\tilde{d}_{1}^{l}(1) & =\frac{\log \left(\tilde{a}^{l}(1) / 39000\right)+\left[\tilde{\delta}^{l}(1)+\left(\tilde{\sigma}^{l}(1)\right)^{2} / 2\right](11 / 365)}{\tilde{\sigma}^{r}(1) \sqrt{11 / 365}} \\
& =\frac{\log (39500 / 39000)+\left[0.02+(0.25)^{2} / 2\right](11 / 365)}{0.25 \sqrt{11 / 365}} \\
& =0.328=d_{1}^{r}(1)
\end{aligned}
$$

with the value of distribution function:

$$
N\left(\tilde{d}_{1}^{l}(1)\right)=N(0.328)=0.6255 .
$$

Now we need $\tilde{d}_{2}^{r}(1)$ :

$$
\tilde{d}_{2}^{r}(1)=d_{1}^{r}(1)-\tilde{\sigma}^{l}(1) \sqrt{\frac{11}{365}}=0.328-0.0434=0.2846
$$

with

$$
N\left(\tilde{d}_{2}^{r}(1)\right)=N(0.2846)=0.61 \text {. }
$$

Substituing these value in $\tilde{c}^{l}(1)$ we have

$$
\tilde{c}^{l}(1)=39500 \cdot 0.6255-39000 \cdot 0.9994 \cdot 0.61=931.524 .
$$

So the value of the call with max belief degree is $\tilde{c}^{l}(1)=$ 931.524.

Now we calculate the minimum value of the call, that is, $\tilde{c}^{l}(0)$, that is

$$
\tilde{c}^{l}(0)=\tilde{a}^{l}(0) N\left(\tilde{d}_{1}^{l}(0)\right)-39000 e^{-(11 / 365) \tilde{\delta}^{l}(0)} N\left(\tilde{d}_{2}^{r}(0)\right)
$$

we need

$$
\begin{aligned}
& \tilde{d}_{1}^{l}(0) \\
& =\frac{\log (\tilde{3} 8000 / 39000)+\left[\tilde{\delta}^{l}(0)+1 / 2\left(\tilde{\sigma}^{l}(0)\right)^{2}\right](11 / 365)}{\tilde{\sigma}^{r}(0) \sqrt{11 / 365}}
\end{aligned}
$$

passing to the numbers we have

$$
\begin{aligned}
\tilde{d}_{1}^{l}(0) & =\frac{\log (38000 / 39000)+\left[0.014+(0.15)^{2} / 2\right](11 / 365)}{0.50 \sqrt{11 / 365}} \\
& =-0.29
\end{aligned}
$$

and we have

$$
N\left(\tilde{d}_{1}^{l}(0)\right)=N(-0.29)=0.3859
$$

now we need also $\tilde{d}_{2}^{r}(0)$ so we have to calculate $d_{1}^{r}(0)$ :

$$
\begin{aligned}
\tilde{d}_{1}^{r}(0) & =\frac{\log \left(\tilde{a}^{r}(y) / 39000\right)+\left[\tilde{\delta}^{r}(y)+\left(\tilde{\sigma}^{r}(y)\right)^{2} / 2\right] 0.03}{\tilde{\sigma}^{l}(y) \sqrt{0.03}} \\
& =1.1686
\end{aligned}
$$

the value of the distribution function is given by

$$
N\left(d_{1}^{r}(0)\right)=N(1.16)=0.50+0.377=0.877 .
$$

Now we calculate $\tilde{d}_{2}^{r}(0)$ :

$$
\tilde{d}_{2}^{r}(0)=\tilde{d}_{1}^{r}(0)-\tilde{\sigma}^{l}(0) \sqrt{\frac{11}{365}}=1.1431,
$$

$$
N\left(\tilde{d}_{2}^{r}(0)\right)=N(1.1431)=0.50+0.3729=0.8729 .
$$

Now we can calculate our minimum value of the call

$$
\begin{aligned}
\tilde{c}^{l}(0) & =\tilde{a}^{l}(0) N\left(\tilde{d}_{1}^{l}(0)\right)-39000 e^{-(11 / 365) \tilde{\delta}^{l}(0)} N\left(\tilde{d}_{2}^{r}(0)\right) \\
& =38000 \cdot 0.3859-39000 \cdot 0.999 \cdot 0.8729 \\
& =-19344.85 .
\end{aligned}
$$


To have the max value of the call, $\tilde{c}^{r}(0)$, we have already $N\left(d_{1}^{r}(0)\right)=0.877$, now we need $N\left(\tilde{d}_{2}^{l}(0)\right)$, where $\tilde{d}_{1}^{l}(0)=$ -0.29 , so

$$
\begin{aligned}
\tilde{d}_{2}^{l}(0) & =\tilde{d}_{1}^{l}(0)-\tilde{\sigma}^{r}(0) \sqrt{\frac{11}{365}} \\
& =-0.29-0.50 \cdot 0.17=-0.3768
\end{aligned}
$$

and $N\left(\tilde{d}_{2}^{l}(0)\right)=N(-0.3768)=0.50-0.1443=0.3557$.

Substituing, we have our max value of the call:

$$
\begin{aligned}
\tilde{c}^{r}(0) & =40000 \cdot N\left(\tilde{d}_{1}^{r}(0)\right)-39000 e^{-t \tilde{\delta}^{r}(0)} N\left(\tilde{d}_{2}^{l}(0)\right) \\
& =40000 \cdot 0.877-39000 \cdot 0.99 \cdot 0.3557=21221.58
\end{aligned}
$$

and our 2006-Black-Scholes triangular fuzzy number is given by

$$
\tilde{c}_{2006}(\omega)= \begin{cases}\frac{\omega-a}{b-a}, & \text { if } \omega \in[a, b] \\ \frac{c-\omega}{c-b}, & \text { if } \omega \in[b, c] \\ 0, & \text { elsewhere }\end{cases}
$$

where $a=\tilde{c}_{2006}^{l}(0)=-19344.85, b=\tilde{c}_{2006}^{l}(1)=931.524$, $c=\tilde{c}_{2006}^{r}(0)=21221.58$.

Therefore, the 2006-Black-Scholes cut functions are given by for all $y \in] 0,1]$

$$
\begin{aligned}
& \tilde{c}_{2006}^{-l}(y)=a+(b-a) y=-19344.85+20276.374 \cdot y \\
& \tilde{c}_{2006}^{-r}(y)=c-(c-b) y=21221.58-20290.056 \cdot y
\end{aligned}
$$

The graph of $\widetilde{c}_{2006}$ looks like a triangle with support: [ $-19344.85,21221.58]$. These evaluations of MIB30 are with the prices of November 11, 2006 when the prices were increased up to 41034 Euro.

Let us see these evaluations also on January 1, 2004 when the MIB30 prices were lower than 2006. So, we take the strike price of the B-S call equal to 27500 and the following cut functions values of initial prices, $\tilde{a}_{2004}^{-l}(0)=26800$; $\tilde{a}_{2004}^{-l}(1)=27000$; and $\tilde{a}_{2004}^{-r}(0)=28000$. Therefore, in the same way of the previous calculus, we have the following cutfunctions values of the B-S fuzzy number $\tilde{c}_{2004}^{l}(0)=-329.90$, $\tilde{c}_{2004}^{-l}(1)=169.64$, and $\tilde{c}_{2004}^{-r}(0)=14933.83$. Indeed, we have

$$
\widetilde{c}_{2004}(\omega)= \begin{cases}\frac{\omega-(-329.9)}{169.64-(-329.9)}, & \text { if } \omega \in[-329.9,169.64] ; \\ \frac{14933.83-\omega}{14933.83-169.64}, & \text { if } \omega \in[169.64,14933.83] ; \\ 0, & \text { elsewhere. }\end{cases}
$$

Now, let us compare the supports of our Black-Scholes fuzzy numbers in these two different dates. On January 1, 2004 the support of $\tilde{c}_{2004}$ was $\Omega_{1}=[-329.90,14933.83]$. On November 11, 2006, the support of $\tilde{c}_{2006}$ was $\Omega_{2}=$
[-19344.85, 21221.58]. So in 2004-2006 the max B-S value increases. But the min decreases:

$$
\begin{gathered}
\tilde{c}_{2004}^{l}(0)=-329.90, \\
\tilde{c}_{2006}^{l}(0)=-19344.85,
\end{gathered}
$$

these negative values can represent indexes of the investor refuse the possibility to buy the stocks of MIB30. The minimum price of the B-S changes from $\tilde{c}_{2004}^{-l}(0)=-329.90$, to $\tilde{c}_{2006}^{l}(0)=-19344.85$ : the investor refuse increased in 2004-2006. So, on November 11, 2006 we could forecast that the market will become with more risks: the risk of loss will increase. The forecast happened, on January 25, 2010 we know that the market loss increases.

The MIB30 is an index of the Italian Stock Exchange so the max of these intervals are indexes of optimistic performance, but the minimum of these B-S intervals are indexes of pessimistic performance on the future. We see that the pessimistic performance becomes more pessimistic.

Further research can be made with Gaussian fuzzy numbers and dynamic program using Matlab.

\section{References}

[1] L. A. Zadeh, "Probability measures of fuzzy events," Journal of Mathematical Analysis and Applications, vol. 23, no. 2, pp. 421427, 1968.

[2] L. Biacino and M. R. Simonelli, "The internal rate of return of fuzzy cash flow," Rivista di Matematica per le Scienze Economiche e Sociali, vol. 14, no. 2, pp. 3-13, 1991.

[3] M. R. Simonelli, "Fuzziness in valuing financial instruments by certainty equivalents," European Journal of Operational Research, vol. 135, no. 2, pp. 296-302, 2001.

[4] M. R. Simonelli, "Statistical fuzzy black-scholes numbers," in Proceedings of the 39th Meeting Euro Working Group on Financial Modelling, Sophia Antipolis, France, 2006.

[5] L. Biacino and A. Lettieri, "Equations with fuzzy numbers," Information Sciences, vol. 47, no. 1, pp. 63-76, 1989.

[6] J. C. Cox and M. Rubinstein, Options Markets, Prentice-Hall, Englewood Cliffs, NJ, USA, 1985.

[7] P. Pianca, Elementi di Teoria delle Opzioni Finanziarie, Giappichelli, Torino, Italy, 2000. 

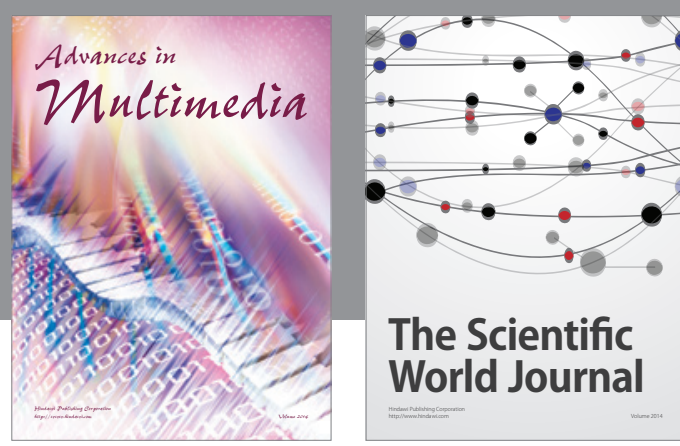

The Scientific World Journal
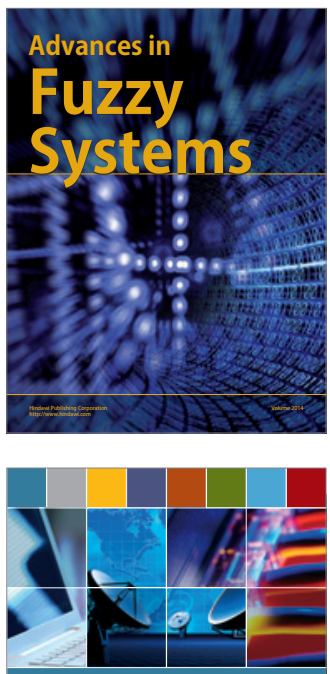

Computer Networks and Communications
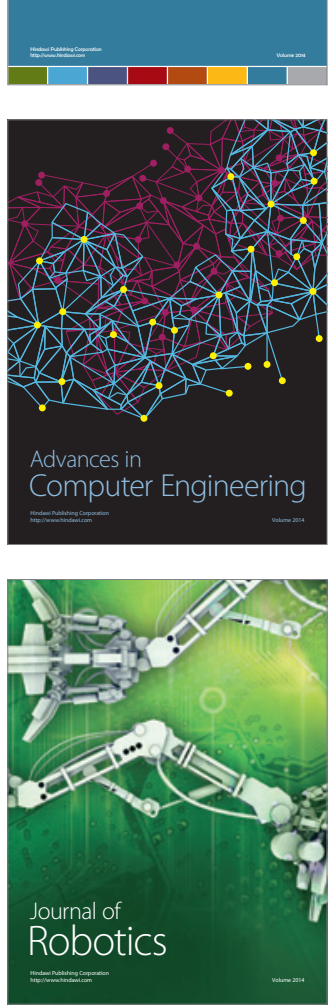
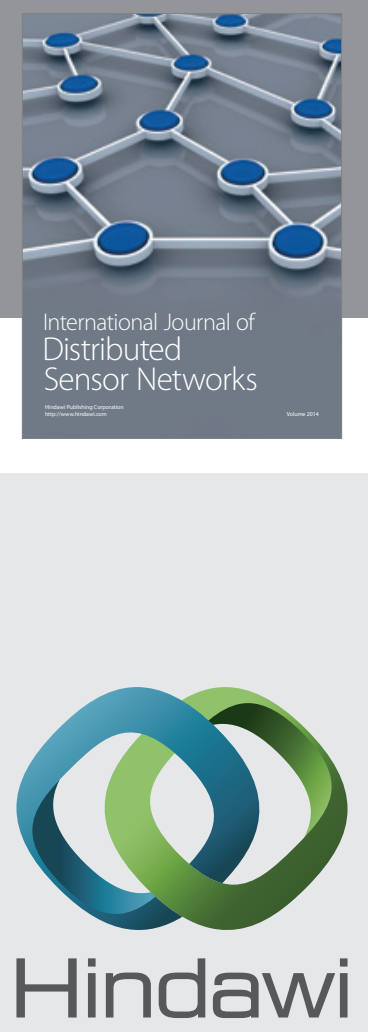

Submit your manuscripts at

http://www.hindawi.com
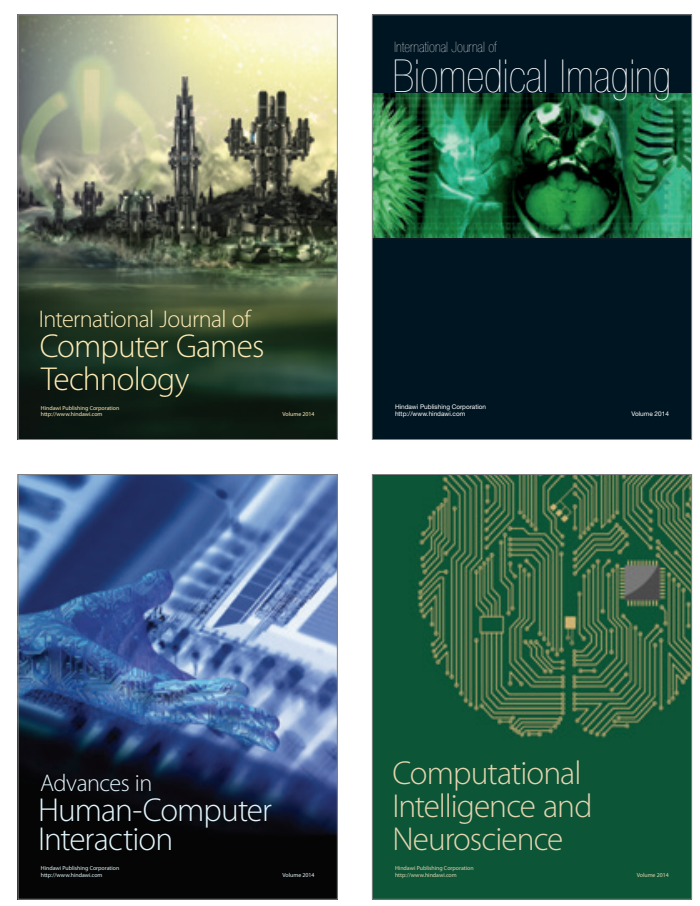
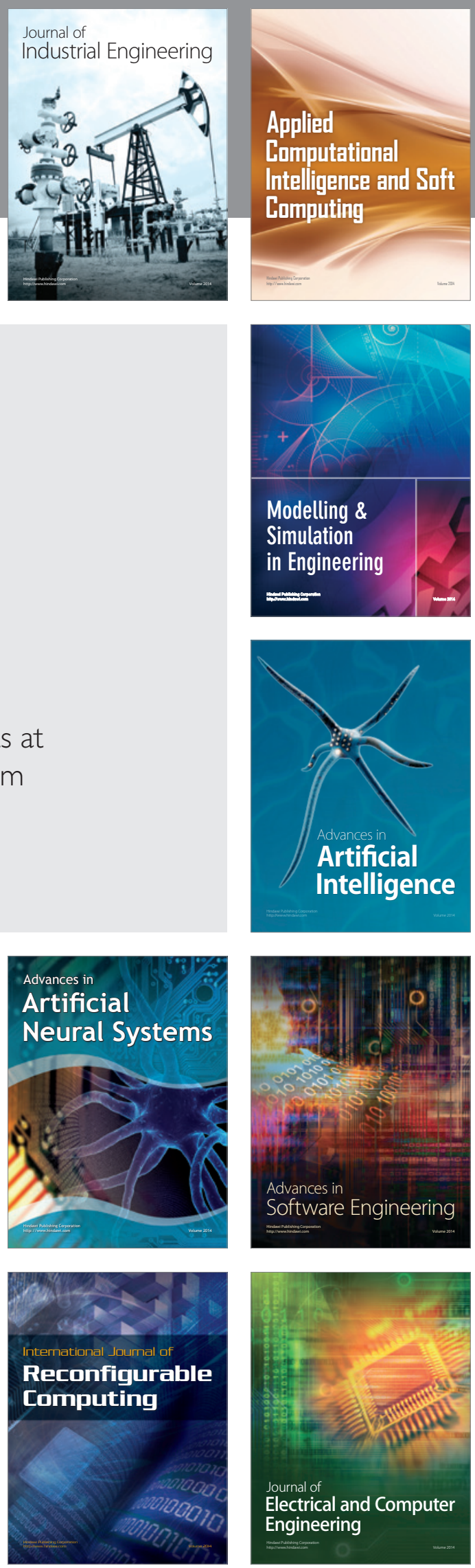